\title{
¿Cuándo sospechar de una trombosis venosa profunda en extremidades superiores?
}

When to suspect of a deep venous thrombosis in the upper limbs?

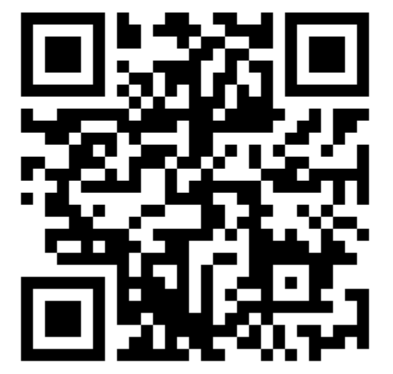

Recibido $14 / 04 / 2021$
${ }^{1}$ Dra. Ana Guiselle Rojas Zúñiga Investigadora independiente, San José, Costa Rica

(iD) https://orcid.org/0000-0002-6696-916X

${ }^{2}$ Dra. Irene María Acuña Núñez Investigadora independiente, San José, Costa Rica

(iD) https://orcid.org/0000-0002-0934-4593

${ }^{3}$ Dra. Daniela Sofía Padilla Elizondo Investigadora independiente, Alajuela, Costa Rica

(iD) https://orcid.org/0000-0003-3950-6095

Corregido $17 / 04 / 2021$
Aceptado $20 / 05 / 2021$

\section{RESUMEN}

La trombosis venosa profunda en extremidades superiores se presenta entre el 4 y $10 \%$ de todos los casos de trombosis venosa profunda, hasta hace algunos años fue considerada una patología rara, dando pie a escasos estudios clínicos aleatorizados, por lo tanto, contribuía a que su diagnóstico fuese poco sospechado. En su gran mayoría el paciente se presenta casi asintomático y con una historia clínica confusa. Los factores de riesgo involucran alteraciones congénitas en la anatomía, así como presencia de malignidad, trombofilias o colocación de implantes trasnvenosos. Las manifestaciones clínicas, los métodos diagnósticos y de tratamiento difieren ampliamente con respecto a la trombosis venosa profunda en extremidades inferiores. Sin embargo, al existir un incremento en el uso de catéteres venosos centrales y la implantación de marcapasos o desfibriladores cardiacos se ha dado de la misma forma un aumento en la manifestación de esta patología. Un diagnóstico y abordaje pronto y certero con terapia anticoagulante son la llave para la prevención complicaciones como el tromboembolismo pulmonar o el síndrome post trombótico.

PALABRAS CLAVE: trombosis venosa profunda; extremidades superiores; diagnóstico; catéter venoso central; anticoagulante.

\section{ABSTRACT}




\begin{abstract}
Deep vein thrombosis in the upper limb is a much less common entity than its lower limb counterpart, it occurs between 4 and $10 \%$ of all cases in regards deep vein thrombosis, a few years ago it was considered a rare pathology, therefore fewer trials were performed, therefore, it contributed to the fact that its diagnosis was less suspected. The vast majority of the patients are almost asymptomatic and they present with a confusing medical history. The risk factors involve congenital alterations in the anatomy as well as the presence of malignancy, thrombophilia or placement of transvenous implants; the symptoms, diagnostic and treatment methods differ widely versus lower extremity deep vein thrombosis. However, as there is an increase in the use of central venous catheters and the implantation of pacemakers or cardiac defibrillators, there has also been an increase in the presence of this pathology. A prompt and accurate diagnosis and approach with anticoagulant therapy are the key to preventing complications such as pulmonary thromboembolism or post-thrombotic syndrome.
\end{abstract}

KEYWORDS: deep vein thrombosis; upper extremities; diagnosis; central venous catheter; anticoagulant.

${ }^{1}$ Médica general, graduada de la Universidad Iberoamérica (UNIBE). cód. MED16699 .Correo: anarz23@gmail.com

${ }^{2}$ Médica general, graduada de la Universidad Iberoamérica (UNIBE). cód. MED16723. Correo: irene.cr@hotmail.com

${ }^{3}$ Médica general, graduada de la Universidad de Iberoamérica (UNIBE), cód. MED16706. Correo: sofia.dpe@gmail.com

\section{INTRODUCCIÓN}

La trombosis venosa profunda en extremidades superiores (TVPES) es una patología un tanto inusual, se describe como la presencia de un trombo el cual obstruye el sistema venoso profundo, fue descrita en 1855 por el patólogo y cirujano ingles Sir James Paget $(1,2)$.

Sin embargo, su presencia en los servicios de urgencia y en pacientes hospitalizados se ha incrementado en los últimos tiempos, principalmente a raíz del aumento en el uso de catéteres venosos centrales y de marcapasos o desfibriladores cardiacos. Publicaciones recientes indican que la incidencia anual de la trombosis venosa profunda (TVP) es de 1/1000 $(3,4)$, de la cual hasta un $10 \%$ corresponde a TVPES $(5,6)$. Se identifica además que un $18 \%$ de las TVP que se diagnostican en pacientes hospitalizados corresponden a la extremidad superior y hasta un $50 \%$ de los pacientes que han presentado una TVPES podría morir en los 5 años posteriores a esta, debido a alguna complicación o malignidad $(5,7)$.

Los métodos diagnósticos en TVPES no están del todo bien establecidos y no cuentan con un algoritmo unificado como si lo tiene la trombosis venosa que se manifiesta en miembro inferior, por esta razón que tanto el proceso diagnóstico como su evolución posterior se tornan un poco complejas.

El tratamiento suele estar basado en controlar los síntomas, la utilización de métodos anticoagulantes, y ocasionalmente la trombolisis, sin embargo, no se tiene claro aún la eficacia del uso de este último y si es posible el manejo únicamente con este fármaco ya que existen pocos estudios que la respalden $(2,8)$.

Por ser una enfermedad con una inusual e infrecuente manera de presentarse, de la cual se cuenta con escaza información que respalde su adecuada evolución, se vuelve todo un reto médico. Por lo tanto, se plantea como objetivo de esta revisión bibliográfica, el brindar conceptos actualizados sobre esta patología, factores de riesgo y 
manifestaciones clínicas que se deben tomar en cuenta ante la sospecha, así como métodos diagnósticos y tratamientos vigentes para un abordaje inmediato.

\section{MÉTODO}

Se realizó una búsqueda de artículos científicos de revistas médicas de las siguientes bases de datos de: PubMed, Medline, Cochrane, Elsevier y Scielo. Se utilizaron un total de 15 publicaciones de países como Estados Unidos, Alemania, Países Bajos, Israel, Inglaterra, entre otros. Los mismos con una antigüedad no mayor a 5 años desde la fecha de su publicación, con el fin de basarse en información reciente, de contenido relevante y vigente. Se enfatizó en la búsqueda los temas de etiopatogenia, factores de riesgo, manifestaciones clínicas, métodos diagnósticos y tratamiento. Se excluyeron todos aquellos artículos con años de publicación mayor a los 5 años, estudios de cohorte, boletines informativos o artículos con un énfasis que no correspondía al buscado con esta revisión.

\section{GENERALIDADES}

En principio la formación del coagulo se fundamenta en un mecanismo protector, que evita el sangrado cuando se presenta una disrupción en la pared de un vaso (9).

La trombosis venosa se define como la presencia de un trombo o coágulo sanguíneo que se origina en el sistema venoso profundo el cual obstruirá de manera parcial o completa el lumen de este, desencadenando sintomatología variada según su localización. Sus presentaciones mas comunes son la trombosis venosa profunda en extremidad inferior (TVPEI) y el tromboembolismo pulmonar (TEP), pero también se manifiestan en miembro superior, venas cerebrales, renales, hepáticas, cavas y mesentéricas $(9,10)$.

La trombosis venosa en extremidades superiores (TVPES) podría ocurrir en cualquier vena del drenaje de miembro superior. Se mencionan como los vasos venosos mas comúnmente afectados por esta patología las venas subclavias $(62 \%)$, las venas axilares $(45 \%)$, y las venas yugulares $(45 \%)(4,10)$.

\section{ETIOPATOGENIA}

Rudolf Virchow describió una triada de elementos presentes al desencadenarse una trombosis venosa profunda, estos son: estasis venosa o turbulencia en el flujo, hipercoagulabilidad sanguínea y disfunción del endotelio los cuales se presentan de maneras variadas para colaborar en la manifestación de la trombosis venosa profunda (11).

Por otro lado, las extremidades superiores presentan características ventajosas que hacen que su incidencia sea mucho menor con respecto a las extremidades inferiores. Entre estas se mencionan: el flujo sanguíneo es muy superior y hay mayor y más continua movilidad del miembro, además no se da el efecto gravitatorio por lo tanto no hay estasis $(10,11)$.

\section{CLASIFICACIÓN}

Tomando en cuenta el postulado anterior se clasifica según su causa en dos grupos: el primario o espontaneo, ya sea idiopático o por el Síndrome de Paget-von Schröetter; y el secundario que en su mayoría se da por malignidad, CVC, dispositivos transvenosos $y$ trombofilias $(7,8)$.

\section{TVPES primario}

En este grupo no se identifica un factor predisponente evidente y se presenta en hasta un $33 \%$ de todas las TVPES $(1,11)$.

Es idiopático cuando se descartan todas las causas posibles, sin embargo, se ha reportado que hasta un $25 \%$ de los pacientes clasificados bajo esta categoría presentan algún tipo de malignidad aun no descubierta (11). 
La trombosis de esfuerzo o Síndrome de Paget-von Schröetter está relacionado a un esfuerzo o movimiento repetitivo que cause hipertrofia en músculos escalenos, muy relacionada a ejercicio excesivo. Produciendo así compromiso de paquete neurovascular, particularmente en la vena subclavia, desencadenando estasis venosa y microtraumas que activan la cascada de coagulación $(1,5)$. Es más frecuente en el brazo dominante, en su mayoría derecho de personas, y en jóvenes. Pintores, deportistas que frecuentan levantar mucho peso, jugadores de tenis, escaladores, nadadores se encuentran entra las actividades que podrían llevar a una trombosis $(3,11)$.

\section{TVPES secundario}

Se incluye en esta categoría todo caso en el que se identifique una causa aparente, es el tipo más frecuente, presentándose en hasta un $80 \%$ de los casos $(10,11)$.

Se desarrolla ante la presencia de cuerpo extraño en el sistema venoso, como la colocación de un catéter venoso central, de un catéter central con inserción periférica, o la implantación de un marcapasos o desfibrilador, estos producen una disrupción del endotelio y por lo tanto se activa cascada de coagulación (6).

\section{FACTORES DE RIESGO}

1. Malignidad: es el factor primario más importante y la segunda causa más común presentándose hasta en un $33 \%$ de los casos (4). Posee una prevalencia de hasta un $64 \%$. Pueden estar ocultos, y se diagnostica durante el seguimiento de pacientes con trombosis. Se mencionan el cáncer de ovario y el adenocarcinoma de pulmón como los más frecuentes. Su presencia se asocia a un estado de hipercoagulabilidad. Asimismo, el paciente con cáncer en estadios avanzados puede cursar con inmovilidad como parte propia de su enfermedad o por efectos adversos al tratamiento $(1,10)$.

Existen además neoplasias, como linfomas o timomas que producen una obstrucción directa en el sistema venoso de miembro superior (10).

2. Catéter venoso central: factor más común y el de mayor presentación en origen secundario, está presente en casi la mitad de los casos de TVSES (4). Se origina por un estado hipercoagulable asociado a una manipulación e irritación de las paredes del endotelio. Dependerá de factores como la técnica, el diámetro, la posición, el tipo de catéter utilizado y cierta medicación insertada por el catéter que tienda a ser viscosa $(4,11)$. Las venas involucradas en este proceso son la yugular interna, la subclavia y la axilar (11).

3. Síndrome de salida torácica: se describe como una anomalía anatómica congénita que presenta un estrechamiento de esta salida la cual es un triángulo conformado por la primera costilla, la clavícula, el tendón del musculo subclavio y el ligamento costoclavicular $(4,12)$. Esta anomalía ocasiona una compresión y consecuentemente daño endotelial de los vasos del drenaje torácico, el cual está conformado por el plexo braquial y la arteria y vena subclavia $(3,8)$.

4. Síndrome de Paget- Schröetter: es una variante venosa del síndrome de salida torácica originalmente descrito en el año 1875 por el inglés James Paget y en 1884 por australiano Leopold van Schrötter, ambos médicos; y posteriormente pronunciado como un síndrome por Hughes, un cirujano ingles en 1949 (1,6). Con una prevalencia de aproximadamente el $30 \%$ de todos los casos de TVPES. Se caracteriza por la presencia trombosis la porción anterior de la salida, específicamente en la vena subclavia en la articulación 
costoclavicular. Provocado por una compresión crónica extrínseca principalmente de la vena subclavia y, pero también de la axilar $(2,3)$. Este es un síndrome poco común y por lo tanto poco estudiando, pero suele presentarse en hombres jóvenes y atléticos, y hasta el momento del diagnóstico, sanos. Con historia de estar realizado actividad física de alto esfuerzo, principalmente hiperabducción o rotación externa de hombro, de manera prolongada $(2,12)$.

5. Otros: a pesar de que los implantes cardiacos como desfibriladores o marcapasos suelen manifestarse como uno de los factores más comunes no se cuenta con estudios recientes al respecto que esclarezcan su prevalencia. Se asocia también a pacientes con síndrome antifosfolipídico, factor $\mathrm{V}$ Leiden y mutaciones en el gen de protrombina. En menor frecuencia se citan cirugías, inmovilización del brazo, trauma, historia de TVPES en los últimos treinta días, uso de anticonceptivos orales y embarazo $(3,5,13)$.

\section{MANIFESTACIONES CLÍNICA}

Se describe un paciente clínicamente asintomático en hasta un $60 \%$ de los portadores (4), razón por la cual es una enfermedad que suele pasar desapercibida al momento de la valoración clínica. Se debe sospechar ante la presencia de síntomas congestivos del sistema venoso por la obstrucción, principalmente de manera unilateral, con dolor agudo que podría llegar a ser severo en brazo, cuello, hombro y región axilar, involucra además inflamación, cianosis, edema, rubor, eritema o sensación de pesadez $(5,11)$. Cuando hay compresión del plexo braquial se asocia debilidad del miembro y parestesias (10). El sistema venoso superficial en el tórax y en miembro superior podrían apreciarse dilatados, conocido como el Signo de Urschel $(2,5)$.
Adicionalmente el paciente con síndrome de Paget - Schrotter acudirá en las primeras 24 horas de iniciado el evento, con historia de ejercicio excesivo en extremidad superior, con sintomatología unilateral que suele presentarse con mayor frecuencia en miembro dominante (12).

En presencia de TVPES el TEP se da en hasta un $10 \%$ (6). En estos casos el paciente se manifiesta con disnea, hemoptisis, dolor torácico, diaforesis y sincope, este último podría ser el síntoma pivote.

También podría ocurrir el síndrome postrombótico, el cual se presenta en casi la mitad de los pacientes con TVPES, con sintomatología algunos años después del evento principal, desarrollándose una insuficiencia venosa por daño valvular desencadenando dolor y edema en el miembro, asociado a prurito y parestesias (5).

\section{DIAGNOSTICO}

- Clínico: la eficacia del examen físico por sí sola no es buena, los signos y síntomas pueden estar ausentes o ser muy variados y poco específicos (13). Los métodos de puntaje poseen una especificidad de $68 \%$, y una sensibilidad de $78 \%$ por lo tanto no se deberían usar para descartar el diagnostico, y se recomienda el uso de imágenes $(6,11)$. Mientras el puntaje de Wells es bien conocido y utilizado en TVP de miembro inferior, el método de puntaje Constans (tabla 1), creado en el 2008 como un test de valoración de probabilidad, se aplica ante la sospecha de TVPES, sin embargo, su efectividad aún no ha sido evaluada $(4,11)$. En este se valora la presencia de cuatro ítems y a los primeros tres se le asigna un punto y al último se le resta, un paciente con cero puntos posee una baja probabilidad mientras que uno con dos o más tiene una posibilidad alta de estar sufriendo 
una trombosis venosa en miembro superior $(13,14)$.

- Pruebas de laboratorio: se utilizan poco, y por si solos no establecen un diagnostico; se aplican las pruebas de coagulación si se sospecha una condición hipercoagulable (5).

Si bien es cierto el dímero $D$ tiene un uso bien conocido en el algoritmo para TVPEI y TEP, en lo que respecta a extremidad superior la bibliografía se encuentra polarizada y no se cuenta con estudios suficientes que determinen su utilidad. Se indica una sensibilidad del $92 \%$ y una especificidad del $60 \%$ utilizando corte de $500 \mu \mathrm{g} / \mathrm{L}$, la cual es similar que en TVPEI (1). Mientras que otros autores prefieren su uso para descartar el diagnostico sin embargo su valor es limitado y tampoco cuenta con suficientes estudios que la respalden $(4,5)$. Se ha descrito también que bajo la regla de dímero $D$ ajustado para la edad aumenta la eficacia del mismo, es decir, que en un paciente menor de 50 años se utiliza el corte anteriormente expuesto, pero para pacientes de mayor edad se realiza la siguiente fórmula para marcar el corte en $\mu \mathrm{g} / \mathrm{L}$ : edad en años por 10 (13).

De manera unánime se prefiere el uso de imágenes radiológicas para determinar 0 confirmar la presencia de trombosis en miembro superior, hasta no contar con más estudios que comprueben la eficacia en el uso de este laboratorio.

\section{DIAGNOSTICO POR IMAGEN RADIOLÓGICA}

En esta radica el diagnóstico de la enfermedad, mostrando una imagen radiológica del trombo intraluminal asociado a una alteración en el flujo venoso. Su propósito es descartar diagnósticos diferenciales como celulitis, hematoma muscular, edema de estasis, linfedema, tromboflebitis superficial, esguince de ligamentos estructurales del hombro, artritis, síndrome postrombótico, y leiomiosarcoma vascular $(2,10)$

- Ultrasonido (US) dúplex por compresión: es considerado el método de primera línea para el diagnóstico. Esta combina la ultrasonografía tradicional y Doppler. En condiciones normales se produce una compresión manual con el cabezal del US sobre la vena y esta colapsa fácilmente, la ausencia del colapso es indicativo de trombosis. Posee una sensibilidad del $97 \%$ y especificidad del $96 \%(4,5,11)$. Tiene varias ventajas sobre otros métodos más sensibles o específicos: es sencillo, portátil y por lo tanto usualmente disponible, además no es invasiva y suele ser económica, sin embargo presenta también desventajas: es operador dependiente, por lo tanto la inexperiencia de este podría omitir un adecuado diagnóstico, y no puede ser utilizada en venas subclavias y braquiocefálicas, por las sombras acústicas que el tejido óseo anexo emite, en estos caos se prefiere usar el US Doppler Color $(4,6)$. EI US Doppler color muestra en una vena sana un característico patrón bifásico de flujo pulsátil, la ausencia de este indica presencia de obstrucción (11).

- Tomografía computarizada y resonancia magnética: ambas técnicas son superiores al ultrasonido, pero suelen utilizarse principalmente en casos en los cuales este no sea concluyente o el paciente tenga contraindicada la venografía con medio de contraste. Presentan la ventaja de poder valorar estructuras anatómicas adyacentes, y por lo tanto identificar obstrucciones causadas por tumoraciones extrínsecas - adenopatías y anormalidades anatómicas como en el caso del Síndrome de salida torácica $(6,11)$. 
- Venografía: es el estándar de oro, tiene una sensibilidad de hasta un 100\% $(3,5,6)$.

Es una técnica de imagen invasiva, en la cual precisa el uso de medio de contraste asociado a radiación rayos $\mathrm{X}$, define de manera detallada la anatomía vascular, así como el adecuado llenado de estas por el medio, haciéndose evidente la falta de este en la vena involucrada. Por la necesidad del medio de contraste iodado es utilizada en casos en donde el US no sea concluyente, ya que podría producir reacciones alérgicas o nefrotoxicidad $(5,11)$.

\section{ALGORITMO DIAGNOSTICO}

Recientemente se ha propuesto un algoritmo para el diagnóstico de TVPES, propuesto por el estudio ARMOUR (imagen 1), sin embargo, su eficiencia aún está en debate (14). Está basado en un estudio prospectivo de 406 pacientes con alta sospecha diagnostica. Primeramente, si la sospecha es alta y el paciente se encuentra en riesgo, es decir, cumple con criterios de malignidad, CVC, presencia de marcapasos o desfibrilador, está hospitalizado y es mayor a 75 años se procede a realizar un US compresivo. Si el paciente no cumple con criterios de riesgo se procede a aplicar el puntaje Constans, en donde a un paciente con un puntaje menor o igual a 1 se le realiza dímero $D$, si está en rangos normales se le da un seguimiento en 3 meses y si es positivo se le realiza un ultrasonido. Por otro lado, si es un paciente con puntaje igual o mayor a dos se le realiza el US compresivo, con tres posibilidades, la confirmación de TVPES, es descarte de trombosis en donde se le dará un seguimiento en tres meses y por último que sea inconcluso en cuyo caso se repetirá en primera instancia el US, si persiste inconcluso se realizara una venografía $(1,5,15)$

Los seguimientos a tres meses permiten que el algoritmo sea seguro de realizar (1).

\begin{tabular}{|c|c|}
\hline Ítem & Puntos \\
\hline Presencia de catéter venoso central o marcapasos & +1 \\
\hline Dolor localizado & +1 \\
\hline Edema unilateral de la extremidad & +1 \\
\hline Sospecha de otro diagnostico igualmente probable & -1 \\
\hline \multicolumn{2}{|c|}{ Clasificación según probabilidad clínica de tres niveles } \\
\hline Baja & $\leq 0$ \\
\hline Intermedia & 1 \\
\hline Alta & $\geq 2$ \\
\hline \multicolumn{2}{|c|}{ Clasificación según probabilidad clínica de dos niveles } \\
\hline TVPES improbable & $\leq 1$ \\
\hline TVPES probable & $\geq 2$ \\
\hline \multicolumn{2}{|c|}{$\begin{array}{l}\text { Fuente. }{ }^{1} \text { Jan Heil, Wolfgang Miesbach, Thomas Vogl, Wolf O. Bechstein, Alexander Reinisch. Deep Vein } \\
\text { Thrombosis of the Upper Extremity A Systematic Review. Dtsch Arztebl Int [Internet]. abril de } 2017 \\
\text { [citado } 14 \text { de abril de 2021];114(14):244-249, }{ }^{2} \text { Noémie Kraaijpoel, Nick van Es, Ettore Porreca, Harry R Büller, } \\
\text { Marcello Di Nisio. The diagnostic management of upper extremity deep vein thrombosis: A review of the literature. } \\
\text { Thromb Res [Internet]. junio de } 2017 \text { [citado } 14 \text { de abril de 2021];156:156:54-59 }\end{array}$} \\
\hline
\end{tabular}




\section{MANEJO}

El objetivo de este se basa en aliviar la sintomatología del paciente, disolver el trombo y a largo plazo evitar recurrencia y complicaciones ya mencionadas, es fundamental establecer el mecanismo causante ya que su manejo estará basado en este $(4,6)$.

Una vez realizado el diagnostico se debe iniciar el tratamiento anticoagulante ya sea oral con warfarina o anticoagulantes directos (ACD), o sistémicos, con heparina de bajo peso molecular o fraccionada $(7,12)$. Si estamos ante un paciente con cáncer se prefiere utilizar heparina de bajo peso molecular (8). La anticoagulación puede utilizarse por sí sola en pacientes con sintomatología leve y con menos de 2 semanas desde inicio de síntomas o asociado a trombólisis en pacientes con sintomatología aguda de moderado a alta intensidad $(6,7,12)$.

Con respecto a los anticoagulantes sistémicos se recomienda el tratamiento por un periodo de 5 días, iniciando con un bolo de heparina de 5000 IU seguido de 15-20 UI/ $\mathrm{kg}$ de peso, y monitorizando con pruebas de coagulación, posteriormente se traslapa a warfarina vía oral por un mínimo de 3 meses y por hasta $6(1,3,6)$.

Con respecto a los $A C D$, se recomienda su uso en pacientes en donde su antecedente causal no sea cáncer (8). Se utiliza una fase de tratamiento inicial con apixaban por 7 días y rivaroxaban por 21 días $(4,16)$.

Por otro lado, los antagonistas de la vitamina $\mathrm{K}$, como la warfarina, han sido empleados para el periodo de mantenimiento, con ajuste de dosis basada en un INR entre 2.0 y 3.0 $(4,8)$.

Con respecto a la trombólisis, lo que se pretende es la disolución del trombo, esta suele utilizarse en casos de TVPES de origen primario y cuando el riesgo de sangrado sea bajo. Los fármacos más habituales son la estreptoquinasa y la alteplasa $(8,16)$.

Existen dos tipos de trombólisis, la sistémica y la mecanofarmacológica, la cual es aplicada por medio de catéter en la vena basilar. Se prefiere este segundo tipo por sobre los sistémicos ya que estos tienen menos riesgo de sangrado, se utiliza menos cantidad de fármaco y se ha reportado menos riesgo a complicaciones $(6,11,12)$.

Se utiliza alteplasa a $0.01 \mathrm{mg} / \mathrm{kg} / \mathrm{h}$ por 24 a $72 \mathrm{~h}$. Se recomienda que su aplicación se realice conjuntamente con anticoagulantes y aspirina $(3,6)$.

Además, no se recomienda su uso en pacientes con más de 14 días de iniciados los síntomas (1).

La trombectomía mecánica percutánea es una técnica recientemente utilizada para remoción del trombo, sin embargo, su efectividad aún no ha sido estudiada $y$ demostrada.

Al paciente con contraindicación para uso de anticoagulantes se tiene como alternativa el uso de filtros percutáneos en vena cava superior, estos son insertados a través de la vena femoral común y colocados a nivel de la vena ácigos $(3,6,8)$.

En el caso de TVPES primario por síndrome de salida torácica, se puede realizar la cirugía descompresiva, la cual consiste en la remoción de la primera costilla y del ligamento costoclavicular para reestablecer el flujo en la vena subclavia $(1,11)$. Este abordaje quirúrgico debe hacerse no antes de hacer trascurrido dos horas de finalizada la trombólisis (6).

En presencia del síndrome de PagetSchröetter, se procurará mejorar la sintomatología asociada a anticoagulación sistémica y trombólisis (15). Sin embargo, a largo plazo se pretende eliminar el mecanismo compresivo que produce la lesión por medio de procedimientos quirúrgicos como venoplastías, trombectomía, reconstrucciones venosas y resección parcial del musculo escaleno. Así 
mismo se usan técnicas endovenosas como la angiografía con balón o "stent" $(2,5,12)$. Se describe hasta que hasta un tercio de los pacientes con este síndrome tendrán una recidiva a pesar del tratamiento $(11,12)$.

En cuestión de cáncer se recomienda la monoterapia anticoagulante con heparina de bajo peso molecular por hasta 6 meses y mientras el tumor persista activo $(11,16)$.

Cuando la causa se asocie a un acceso venoso se iniciará inmediatamente con anticoagulantes sistémicos y se establecerá la verdadera necesidad del uso de este tipo de vía, si se determina que este debe de utilizarse con obligatoriedad deberá entonces estar adecuadamente colocado, funcionando y libre de infección, además se mantiene con anticoagulantes por hasta 6 meses $(1,11,12)$.

\section{CONCLUSIONES}

Al ser una entidad un poco inusual, la trombosis venosa profunda en miembro superior suele ser poco sospechada, además el conocimiento e investigaciones que existe sobre esta son limitados. Sin embargo, presenta un aumento significativo en su incidencia en los últimos años debido a las razones mencionadas, por lo tanto, su reconocimiento temprano y certero es primordial para una pronta atención y por lo tanto prevenir complicaciones para el paciente.

Es de suma importancia reconocer los principales factores de riesgo tanto primarios como secundarios y así tener la sospecha ante la presencia de su muy variada sintomatología. Se debe sospechar estar ante la presencia del síndrome de Paget -
Schröetter en un paciente masculino, joven, deportista que consulta a un servicio de emergencias por dolor, edema, rubor en uno de sus brazos, asociado a parestesias.

También se debe sospechar en pacientes hospitalizados en los que se esté utilizando un acceso venoso central o en un paciente al que recientemente se le coloca un implante cardiaco y que consulte con sintomatología unilateral.

Una vez se valore la probabilidad de la TVPES se recomienda iniciar sistemáticamente el protocolo diagnóstico, empleando las herramientas recomendadas como la puntuación de Constans, posteriormente la valoración del dímero $D$ y la utilización de métodos de imagen, principalmente y como primera opción la ultrasonografía por compresión, que en la mayoría de los casos confirmará el diagnostico.

Por último, el abordaje se inicia con anticoagulación ya sea por vía oral o sistémica, según las características del paciente y la causa que lo llevo a TVPES. Se recomienda extender el tratamiento con warfarina por un mínimo de tres meses. Así mismo, se valora la viabilidad de realizar trombolisis con estreptoquinasa y alteplasa, la cual depende de la severidad de los síntomas y el riesgo de sangrado que presente el paciente. Ante un caso de trombosis secundaria a catéter venoso central se estudia la verdadera de necesidad de la permanencia de este, en donde el beneficio debe superar al riesgo.

\section{Los autores declaran no tener conflicto de interés.}


Figura 1. Algoritmo diagnostico para trombosis venosa profunda en miembro superior basado en estudio ARMOUR

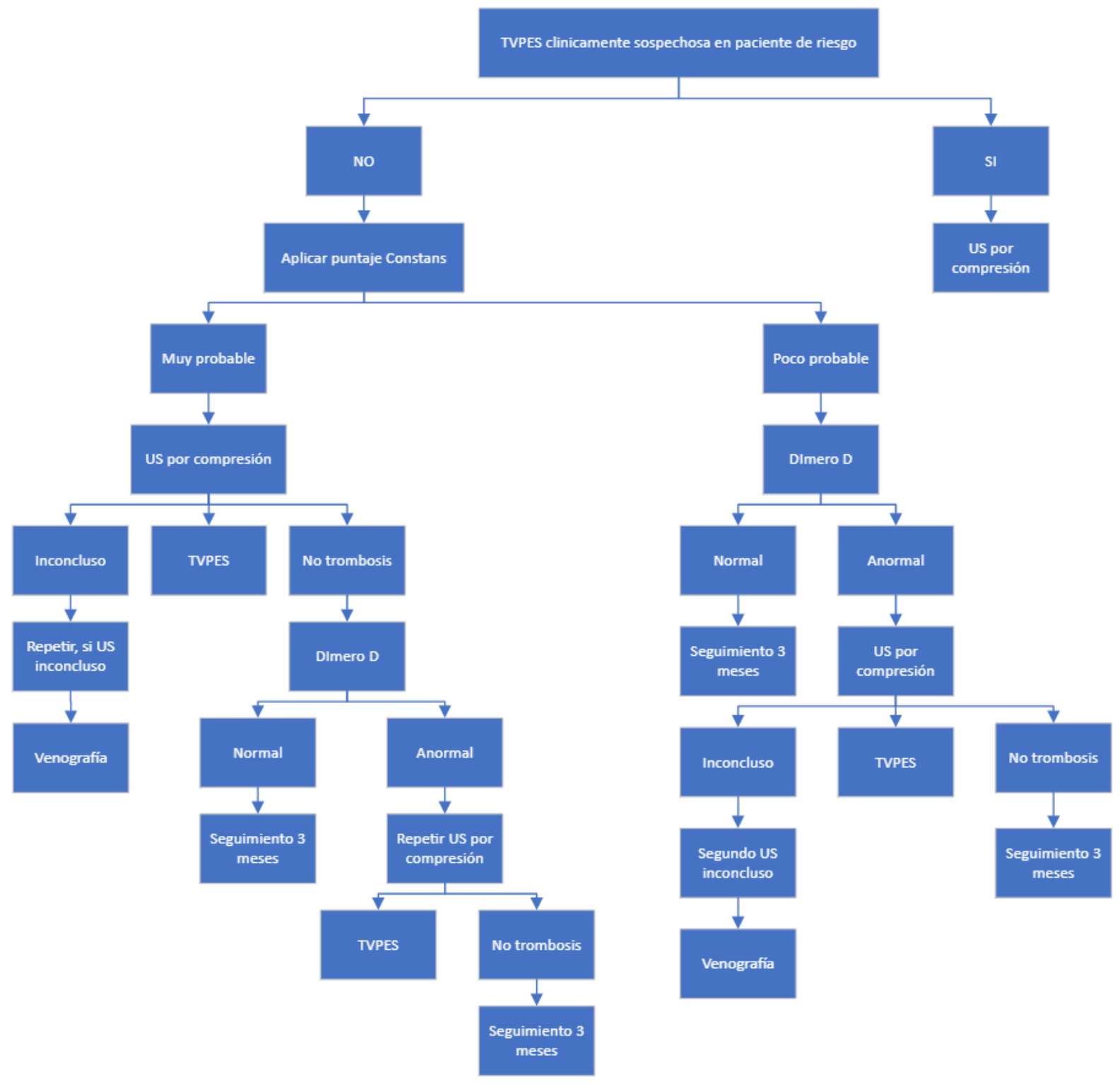

Fuente. ${ }^{1}$ Extremity Deep Vein Thrombosis: Symptoms, Diagnosis, and Treatment. Isr Med Assoc J [Internet]. enero de 2018 [citado 14 de abril de 2021];20(1):53-7.

${ }^{2} \mathrm{~N}$. van Es, S. M. Bleker, M. Di Nisio, A. Kleinjan, J. Beyer-Westendorf. Improving the diagnostic management of upper extremity deep vein thrombosis. J Thromb Haemost [Internet]. octubre de 2016 [citado 14 de abril de 2021];15:66-73. 


\section{REFERENCIAS}

1. Floris $T$ M Bosch, Marcello Di Nisio, Harry R Büller, Nick van Es. Diagnostic and Therapeutic Management of Upper Extremity Deep Vein Thrombosis. J Clin Med [Internet]. julio de 2020 [citado 14 de abril de 2021];9(7):2069. Disponible en:

https://www.ncbi.nlm.nih.gov/pmc/articles/PMC74 08847/\#B35-jcm-09-02069

2. Jorge Carmelo Martínez-Gil, Hubert BahamonRivera, Silvana Jimenez-Salazar, Kenny BuitragoToro, Ana María Baldovino-Chiquillo, Jaime Andrés-Arango. Trombosis venosa de sitio inusual: síndrome de Paget-Schroetter. AMC [Internet]. junio de 2019 [citado 14 de abril de 2021];23(3):374-81. Disponible en: http://scielo.sld.cu/scielo.php?script=sci isoref\&pi $\mathrm{d}=\mathrm{S} 1025-02552019000300374 \&$ Ing=es\&tlng=es

3. Mark Porteous, Jecko Thachil. When deep vein thrombosis occurs in the upper limb. Br J Hosp Med (Lond) [Internet]. agosto de 2016 [citado 14 de abril de 2021];77(8):448-53. Disponible en: https://pubmed.ncbi.nlm.nih.gov/27487054/

4. Jan Heil, Wolfgang Miesbach, Thomas Vogl, Wolf O. Bechstein, Alexander Reinisch. Deep Vein Thrombosis of the Upper Extremity A Systematic Review. Dtsch Arztebl Int [Internet]. abril de 2017 [citado 14 de abril de 2021];114(14):244-249. Disponible

en: https://www.ncbi.nlm.nih.gov/pmc/articles/PMC54 15909/

5. Areg Grigorian, Jeffry T. Nahmias. Upper Extremity Deep Venous Thrombosis. StatPearls [Internet]. 26 de julio de 2020 [citado 14 de abril de 2021]; Disponible en: https://www.ncbi.nlm.nih.gov/books/NBK482420/ $\# !$ po $=95.0000$

6. Jaber Mustafa, Ilan Asher, Zev Sthoeger. Upper Extremity Deep Vein Thrombosis: Symptoms, Diagnosis, and Treatment. Isr Med Assoc J [Internet]. enero de 2018 [citado 14 de abril de 2021];20(1):53-7. Disponible en: https://pubmed.ncbi.nlm.nih.gov/29658209/

7. Joshua Feinberg, Emil Eik Nielsen, Janus C Jakobsen. Thrombolysis for acute upper extremity deep vein thrombosis (Review). Cochrane Database of Systematic Reviews [Internet]. 2017 [citado 14 de abril de 2021];12. Disponible en: https://www.cochranelibrary.com/cdsr/doi/10.100 2/14651858.CD012175.pub2/full/es\#CD012175abs-0002

8. Timothy A. Carlon, Deepak Sudheendra. Interventional Therapy for Upper Extremity Deep Vein Thrombosis. Semin Intervent Radiol [Internet]. 2017 [citado 14 de abril de 2021];34(1):54-60. Disponible en:
https://www.ncbi.nlm.nih.gov/pmc/articles/PMC53 34483/

9. Sheikh M. Waheed, Pujitha Kudaravalli, David T. Hotwagner. Deep Vein Thrombosis. StatPearls [Internet]. 10 de agosto de 2020 [citado 14 de abril de 2021]; Disponible en: https://www.ncbi.nlm.nih.gov/books/NBK507708/

10. Trombosis venosa profunda de miembros superiores. CCM [Internet]. 2020 [citado 14 de abril de 2021];24(4):1226-44. Disponible en: https://www.medigraphic.com/cgibin/new/resumen.cgi?IDARTICULO=98402

11. Adam M Noyes, John Dickey. The Arm is Not the Leg: Pathophysiology, Diagnosis, and Management of Upper Extremity Deep Vein Thrombosis. R I Med J (2013) [Internet]. mayo de 2017 [citado 14 de abril de 2021];100(5):33-6. Disponible en: https://pubmed.ncbi.nlm.nih.gov/28459919/

12. Patrick Hangge, Lisa Rotellini-Coltvet, Amy R. Deipolyi, Hassan Albadawi, Rahmi Oklu. PagetSchroetter syndrome: treatment of venous thrombosis and outcomes. Cardiovasc Diagn Ther [Internet]. diciembre de 2017 [citado 14 de abril de 2021];7(3):S285-90. Disponible en: https://www.ncbi.nlm.nih.gov/pmc/articles/PMC57 78512/

13. N. van Es, S. M. Bleker, M. Di Nisio, A. Kleinjan, J. Beyer-Westendorf. Improving the diagnostic management of upper extremity deep vein thrombosis. J Thromb Haemost [Internet]. octubre de 2016 [citado 14 de abril de 2021];15:66-73. Disponible

en: https://onlinelibrary.wiley.com/doi/full/10.1111/jth. $\underline{13536}$

14. Noémie Kraaijpoel, Nick van Es, Ettore Porreca, Harry R Büller, Marcello Di Nisio. The diagnostic management of upper extremity deep vein thrombosis: A review of the literature. Thromb Res [Internet]. junio de 2017 [citado 14 de abril de 2021];156:156:54-59. Disponible en: https://pubmed.ncbi.nlm.nih.gov/28586697/

15. Lauren P Cote, Steven Greenberg, Joseph A Caprini. Comparisons Between Upper and Lower Extremity Deep Vein Thrombosis: A Review of the RIETE Registry. Clin Appl Thromb Hemost [Internet]. octubre de 2017 [citado 14 de abril de 2021];23(7):748-54. Disponible en: https://pubmed.ncbi.nlm.nih.gov/27572888/

16. Francisco Sánchez Montiel, Raein Ghazvinian, Anders Gottsäter, Johan Elf. Treatment with direct oral anticoagulants in patients with upper extremity deep vein thrombosis. Thromb J [Internet]. octubre de 2017 [citado 14 de abril de 2021];15(26). Disponible en: https://www.ncbi.nlm.nih.gov/pmc/articles/PMC56 25705/ 